\title{
SHEAVES OF NONCOMMUTATIVE ALGEBRAS AND THE BEILINSON-BERNSTEIN EQUIVALENCE OF CATEGORIES
}

\author{
T. J. HODGES AND S. P. SMITH
}

\begin{abstract}
Let $X$ be an irreducible algebraic variety defined over a field $k$, let $\mathscr{R}$ be a sheaf of (noncommutative) noetherian $k$-algebras on $X$ containing the sheaf of regular functions $\mathcal{O}$ and let $R$ be the ring of global sections. We show that under quite reasonable abstract hypotheses (concerning the existence of a faithfully flat overring of $R$ obtained from the local sections of $\mathscr{R}$ ) there is an equivalence between the category of $R$-modules and the category of sheaves of $\mathscr{R}$-modules which are quasicoherent as $\boldsymbol{O}$-modules. This shows that the equivalence of categories established by Beilinson and Bernstein as the first step in their proof of the KazhdanLusztig conjectures (where $R$ is a primitive factor ring of the enveloping algebra of a complex semisimple Lie algebra, and $\mathscr{R}$ is a sheaf of twisted differential operators on

- a generalised flag variety) is valid for more fundamental reasons than is apparent from their work.
\end{abstract}

\section{Introduction.}

1.1. This paper is motivated by the Beilinson-Bernstein Theorem [BB]. Very briefly, this says that for certain primitive factor rings $D_{\lambda}$ of the enveloping algebra of a complex semisimple Lie algebra there is an associated sheaf $\mathscr{D}_{\lambda}$ of noncommutative algebras over a complex projective algebraic variety $X$ such that $D_{\lambda}=\Gamma\left(X, \mathscr{D}_{\lambda}\right)$ and there is an equivalence between the category of left $D_{\lambda}$-modules and the category of sheaves of left $\mathscr{D}_{\lambda}$-modules which are quasicoherent as $\mathcal{O}$-modules $(\mathcal{O}$ is the structure sheaf of $X$, and is a subsheaf of $\mathscr{D}_{\lambda}$ ). In this paper we abstract some of the essential features of their construction in an attempt to understand what ring theoretic properties of $D_{\lambda}$ ensure this equivalence of categories. We show that the equivalence of categories follows from the existence of a faithfully flat overring of $D_{\lambda}$.

Let us give the details.

1.2. Let $k$ be any field and $X$ an irreducible algebraic variety over $k$. Let $\mathscr{R}$ be a sheaf of noncommutative noetherian $k$-algebras over $X$. Set $R=\Gamma(X, \mathscr{R})$ and suppose that $R$ has a classical ring of quotients $Q$. If $U$ is an open affine subset of $X$ write $R_{U}=\Gamma(U, \mathscr{R})$. We make the following assumptions concerning $\mathscr{R}$ from which the Theorem below will be deduced.

(i) The structure sheaf $\mathcal{O}$ of $X$ is a subsheaf of $\mathscr{R}$ and $\mathscr{R}$ is a quasicoherent sheaf of left $\mathcal{O}$-modules.

Received by the editors November 29, 1983.

1980 Mathematics Subject Classification. Primary 16A08; Secondary 14A10, 16A33, 16A63. 
(ii) If $U \subset X$ is an open affine subset of $X$, then $R_{U}$ is a subalgebra of $Q$ containing $R$ and is generated as a right or left $R$-module by $\Gamma(U, \mathcal{O})$.

(iii) There is a finite open affine cover $\left(U_{\alpha}\right)$ for $X$ such that the diagonal embedding $R \rightarrow \oplus R_{\alpha}$ (where $R_{\alpha}=\Gamma\left(U_{\alpha}, \mathscr{R}\right)$ ) obtained from the restriction maps makes $\oplus R_{\alpha}$ a faithfully flat right $R$-module.

Write $R$-Mod for the category of left $R$-modules and $\mathscr{R}-\mathscr{M} o d$ for the category of sheaves of left $\mathscr{R}$-modules which are quasicoherent as $\mathcal{O}$-modules.

THEOREM. There is an equivalence between $R$-Mod and $\mathscr{R}-\mathscr{M}$ od given by the mutually inverse functors $M \rightarrow \mathscr{R} \otimes_{R} M$ and $\mathscr{M} \rightarrow \Gamma(X, \mathscr{M})$.

1.3. It is straightforward to check that $\mathscr{D}_{\lambda}$ satisfies (i) and (ii) above. Although (iii) follows from the equivalence of categories established in [BB] we have not been able to establish its truth on a priori grounds except in some special cases. However, there are reasons for expecting this may be possible (see \$3.1) and then the Theorem would imply the result of Beilinson and Bernstein. Joseph and Stafford [JS] have given a direct proof of the flatness condition in (iii), so one only needs to find a direct proof of the "faithfulness" condition.

1.4. This paper is primarily written for noncommutative ring theorists. The paper can be read without a knowledge of the work of Beilinson and Bernstein, but we expect that an understanding of their construction would make the work here more meaningful.

\section{Proof of the Theorem.}

2.1. The Theorem will be proved using the language of torsion theory. We recall the standard terminology and results which we require. The reader is refered to [St] for a thorough treatment and proofs.

Let $R$ be a ring and $A$ a ring containing $R$ (with the same identity). If $A$ is flat as a right $R$-module and $A \otimes_{R} A \simeq A$ (under the map $a \otimes b \rightarrow a b$ ) as an $A$ - $A$ bimodule we call $A$ a perfect (left) localisation of $R$. Denote by $\mathscr{T}$ the class of all left $R$-modules $M$ satisfying $A \otimes_{R} M=0$. We call $\mathscr{T}$ the torsion class associated to $A$. Because $A_{R}$ is flat, the class $\mathscr{T}$ is closed under submodules, factor modules and extensions. If $M$ is an $R$-module there is a largest submodule of $M$ contained in $\mathscr{T}$; we denote this submodule by $\tau(M)$ and call it the torsion submodule of $M$. If $\tau(M)=M$ we call $M$ a torsion module, and if $\tau(M)=0$ we say that $M$ is torsion-free. Denote by $\mathscr{F}$ the class of left ideals $I$ of $R$ such that $R / I$ is a torsion module. We call $\mathscr{F}$ the Gabriel filter associated to $A$.

If $a \in A$, there exists an $I$ in $\mathscr{F}$ such that $I a \subset R$. If $M$ is an $R$-module, then $\tau(M)=\{m \in M \mid \operatorname{Im}=0$ for some $I$ in $\mathscr{F}\}$. The kernel of the natural map $\phi$ : $M \rightarrow A \otimes_{R} M$ is $\tau(M)$ and $A \otimes M / \phi(M)$ is a torsion module. If $I$ and $J$ are in $\mathscr{F}$ so are $I \cap J$ and $I J$. The filter $\mathscr{F}$ may be characterized as those left ideals $I$ of $R$ such that $A I=A$. These facts will be used without comment in what follows.

2.2. Consider the situation described in $\$ 1.2$. For simplicity we shall write $U_{\alpha_{1} \cdots \alpha_{n}}=U_{\alpha_{1}} \cap \cdots \cap U_{\alpha_{n}}$, and $R_{\alpha_{1} \cdots \alpha_{n}}=\Gamma\left(U_{\alpha_{1} \cdots \alpha_{n}}, \mathscr{R}\right)$. If $\mathscr{M}$ is a sheaf of $\mathscr{R}$-modules we shall write $M=\Gamma(X, \mathscr{M})$ and $M_{\alpha_{1} \cdots \alpha_{n}}=\Gamma\left(U_{\alpha_{1} \cdots \alpha_{n}}, \mathscr{M}\right)$. Abusing notation, we shall write $\rho_{\alpha_{1} \cdots \alpha_{n}}$ for the restriction map $M_{\beta_{1} \cdots \beta_{m}} \rightarrow M_{\alpha_{1} \cdots \alpha_{n}}$ for any subset 
$\left\{\beta_{1}, \ldots, \beta_{m}\right\} \subset\left\{\alpha_{1}, \ldots, \alpha_{n}\right\}$. No confusion should arise as the domain of $\rho_{\alpha_{1} \cdots \alpha_{n}}$ will always be clear from the context.

The stalk of $\mathscr{R}$ at $x \in X$ will be denoted by $R_{x}$. If $V \subset X$ is an open subset, denote $\Gamma(V, \mathcal{O})$ by $A_{V}$, and write $A_{x}$ for the stalk of $\mathcal{O}$ at $x$.

2.3. Let us point out some immediate consequences of the assumptions made in $\S 1.2$.

The quasicoherence of $\mathscr{R}$ ensures that if $U \subset V$ are open affine subsets of $X$, then $R_{U}=A_{U} \otimes_{A_{V}} R_{V}$. If $V \subset X$ is open affine, then there is an equivalence between the categories $R_{V}$ Mod (of left $R_{V}$-modules) and $\left.\mathscr{R}\right|_{V}=\mathscr{M}_{o d}$ (of sheaves of left $\left.\mathscr{R}\right|_{V}$-modules which are quasicoherent as $\left.\mathcal{O}\right|_{V}$-modules). If $M$ is an $R_{V}$-module, then view $M$ as an $A_{V}$-module and form the sheaf $\tilde{M}$ (the notation is that of $[\mathbf{H}]$ ). If $U \subset V$ is an open affine subset, then

$$
\Gamma(U, \tilde{M})=A_{U} \otimes_{A_{V}} M=A_{U} \otimes_{A_{V}}\left(R_{V} \otimes_{R_{V}} M\right)=R_{U} \otimes_{R_{V}} M,
$$

so $\tilde{M}$ is a sheaf of $\left.\mathscr{R}\right|_{V}$-modules with global sections $M$. Also, if $\left.\mathscr{M} \in \mathscr{R}\right|_{V}-\mathscr{M}_{o d}$, then $\mathscr{M} \simeq \Gamma(V, \mathscr{M}) \tilde{\text { and }} \Gamma(V, \mathscr{M})$ is an $R_{V}$-module. Hence the equivalence between $R_{V}$ Mod and $\left.\mathscr{R}\right|_{V} \mathscr{M}$ od.

2.4. It is not strictly necessary to assume that $R$ has a classical ring of fractions $Q$. One could replace $Q$ in the hypotheses by the maximal flat epimorphic extension of $R$. The existence of $Q$ is only used in applying the following result.

Proposition [St, Chapter XI, Proposition 2.4]. If $A$ is a ring with $R \subset A \subset Q$ and $A_{R}$ is flat, then $A$ is a perfect left localisation of $R$.

COROLLARY. If $U \subset U_{\alpha}$ and $U$ is open affine, then $R_{U}$ is a perfect left localisation of $R$.

Proof. By assumption, $R_{\alpha}$ is flat as a right $R$-module, so is a perfect left localisation by the Proposition.

Put $A_{\alpha}=\Gamma\left(U_{\alpha}, \mathcal{O}\right)$. As $\mathscr{R}$ is quasicoherent, $R_{U}=A_{U} \otimes_{A_{\alpha}} R_{\alpha}$. Now, as $A_{U}$ is flat as an $A_{\alpha}$-module, $R_{U}$ is flat as a right $R_{\alpha}$-module. Hence $R_{U}$ is flat as a right $R$-module. Apply the Proposition to $R_{U}$.

Thus the hypotheses in $\$ 1.2$ ensure that each $R_{\alpha_{1} \cdots \alpha_{n}}$ is a perfect left localisation of $R$. We shall denote the torsion class associated to the perfect left localisation $R_{\alpha}$ of $R$ by $\mathscr{T}_{\alpha}$. The associated Gabriel filter will be denoted by $\mathscr{F}_{\alpha}$, and the associated torsion functor will be denoted by $\tau_{\alpha}$.

2.5. LemMA. Let $R$ be a noetherian ring with ring of fractions $Q$. Let $A_{1}$ and $A_{2}$ be perfect left localisations of $R$. Suppose that each $A_{i}$ is generated as either a right or left $R$-module by a subset $S_{i}$ and that the elements of $S_{1}$ commute with the elements of $S_{2}$. Let $B$ denote the subring of $Q$ generated by $A_{1}$ and $A_{2}$. Then there is an isomorphism of $A_{1}-A_{2}$ bimodules $A_{1} \otimes_{R} A_{2} \rightarrow B$ given by $a_{1} \otimes a_{2} \rightarrow a_{1} a_{2}$, and hence there is an isomorphism of $R-R$ bimodules $A_{1} \otimes_{R} A_{2} \simeq A_{2} \otimes_{R} A_{1}$.

Proof. If $A$ is a perfect left localisation of $R$, the map $A \otimes_{R} A \rightarrow A$ given by $a \otimes a^{\prime} \rightarrow a a^{\prime}$ is an $A-A$ bimodule isomorphism.

The natural maps

$$
A_{1} \otimes_{R} B \rightarrow A_{1} \otimes_{R}\left(A_{1} \otimes_{A_{1}} B\right) \rightarrow\left(A_{1} \otimes_{R} A_{1}\right) \otimes_{A_{1}} B \rightarrow A_{1} \otimes_{A_{1}} B \rightarrow B
$$


are all isomorphisms of $A_{1}-B$ bimodules, so the composition gives $A_{1} \otimes_{R} B \simeq B$ by $a \otimes b \rightarrow a b$.

Tensor the exact sequence $0 \rightarrow A_{2} \rightarrow B \rightarrow B / A_{2} \rightarrow 0$ with the flat $R$-module $A_{1}$. To prove the first part of the lemma it is enough to prove that $A_{1} \otimes_{R}\left(B / A_{2}\right)=0$, because one may then compose the isomorphisms $A_{1} \otimes_{R} A_{2} \rightarrow A_{1} \otimes_{R} B \rightarrow B$ to get the result.

A typical element of $B$ is a finite sum of terms of the form $a_{1} a_{2}$ with $a_{1} \in A_{1}$ and $a_{2} \in A_{2}$ (just use the fact that $S_{1}$ and $S_{2}$ commute, and that $A_{1}=S_{1} R=R S_{1}$ ). Let $\mathscr{F}$ denote the Gabriel filter associated to $A_{1}$. Given $a_{1} \in A_{1}, a_{2} \in A_{2}$ pick $I$ in $\mathscr{F}$ such that $I a_{1} \subset R$; whence $I a_{1} a_{2} \subset A_{2}$. So the image of $a_{1} a_{2}$ in $B / A_{2}$ is in the torsion submodule (associated to $A_{1}$ ). As every element of $B / A_{2}$ is a finite sum of torsion elements, $B / A_{2}$ is itself torsion. In other words $A_{1} \otimes_{R}\left(B / A_{2}\right)=0$.

We have shown $A_{1} \otimes_{R} A_{2} \simeq B$ as $A_{1}-A_{2}$ bimodules, hence as $R$ - $R$ bimodules. Reversing the roles of $A_{2}$ and $A_{1}$, we also have $A_{2} \otimes_{R} A_{1} \simeq B$ as $R-R$ bimodules. The lemma follows.

In the commutative case the isomorphism $A_{1} \otimes_{R} A_{2} \rightarrow A_{2} \otimes_{R} A_{1}$ would just be $a_{1} \otimes a_{2} \rightarrow a_{2} \otimes a_{1}$. In the noncommutative case the map is not so clear; first $a_{1} \otimes a_{2}$ is mapped to $a_{1} a_{2} \in B$, then $a_{1} a_{2}$ is expressed as a sum of terms of the form $a_{2}^{\prime} a_{1}^{\prime}$ with $a_{2}^{\prime} \in A_{2}, a_{1}^{\prime} \in A_{1}$ and each $a_{2}^{\prime} a_{1}^{\prime}$ is mapped to $a_{2}^{\prime} \otimes a_{1}^{\prime} \in A_{2} \otimes A_{1}$.

2.6. Lemma. $R_{\alpha_{1}} \otimes_{R} \cdots \otimes_{R} R_{\alpha_{n}} \simeq R_{\alpha_{1} \cdots \alpha_{n}}$ as $R_{\alpha_{1}}-R_{\alpha_{n}}$ bimodules.

PROOF. For each $m \leqslant n, R_{\alpha_{1} \cdots \alpha_{m}}$ is a perfect left localization of $R$ (as remarked in $\S 2.4)$. Now apply Lemma 2.5 inductively. Notice that to apply 2.5 one must use the fact that $\Gamma\left(U_{\alpha} \cap U_{\beta}, \mathcal{O}\right)$ is generated by the images (under the restriction maps) of $\Gamma\left(U_{\alpha}, \mathcal{O}\right)$ and $\Gamma\left(U_{\beta}, \mathcal{O}\right)$, and this is guaranteed by the assumption that $X$ is a variety.

2.7. LeMMA. Let $N$ be an $R_{\alpha_{1} \cdots \alpha_{n}}$-module such that $R_{\alpha_{1} \cdots \alpha_{n+1}} \otimes N=0$, where the tensor product is over $R_{\alpha_{1} \cdots \alpha_{n}}$. Then $R_{\alpha_{n+1}} \otimes_{R} N=0$.

ProOF.

$$
R_{\alpha_{n+1}} \otimes_{R} N=R_{\alpha_{n+1}} \otimes_{R}\left(R_{\alpha_{1} \cdots \alpha_{n}} \otimes N\right)=\left(R_{\alpha_{n+1}} \otimes_{R} R_{\alpha_{1} \cdots \alpha_{n}}\right) \otimes N,
$$

where the second tensor product is over $R_{\alpha_{1} \cdots \alpha_{n}}$. Now apply the previous lemma.

2.8. Lemma. Let $\mathscr{M} \in \mathscr{R}-\mathscr{M}$ od and put $M=\Gamma(X, \mathscr{M})$. If $\rho_{\alpha}: M \rightarrow M_{\alpha}$ is the restriction map, then $\operatorname{ker} \rho_{\alpha}=\tau_{\alpha}(M)$.

Proof. If $m \in \tau_{\alpha}(M)$, then $I m=0$ for some $I \in \mathscr{F}_{\alpha}$. Hence

$$
\rho_{\alpha}(m) \in R_{\alpha} \rho_{\alpha}(m)=R_{\alpha} I \rho_{\alpha}(m)=R_{\alpha} \rho_{\alpha}(I m)=0 .
$$

So $\tau_{\alpha}(M) \subset \operatorname{ker} \rho_{\alpha}$.

Put $N=\operatorname{ker} \rho_{\alpha}$, and $N_{\beta}=\rho_{\beta}(N)$. As $\mathscr{R}$ is quasicoherent, $\rho_{\alpha \beta}\left(N_{\beta}\right)=R_{\alpha \beta} \otimes_{R_{\beta}} N_{\beta}$. But $\rho_{\alpha \beta}\left(N_{\beta}\right)=\rho_{\alpha \beta} \rho_{\beta}(N)=\rho_{\alpha \beta} \rho_{\alpha}(N)=0$. Hence, by the previous lemma, $R_{\alpha} \otimes_{R} N_{\beta}$ $=0$, and so each $N_{\beta} \in \mathscr{T}_{\alpha}$.

Pick $n \in N$, and put $n_{\beta}=\left.n\right|_{U_{\beta}}$. By the previous paragraph, for each $\beta$ there exists $I_{\beta} \in \mathscr{F}_{\alpha}$ with $I_{\beta} n_{\beta}=0$. Put $I=\bigcap_{\beta} I_{\beta}$. Thus $I \in \mathscr{F}_{\alpha}$ and $I n_{\beta}=0$ for all $\beta$. But $\operatorname{In}_{\beta}=\left.\operatorname{In}\right|_{U_{\beta}}$; as $I n$ is locally zero and the $U_{\beta}$ cover $X$, In is globally zero. That is In $=0$, and hence $n \in \tau_{\alpha}(M)$. 
2.9. Lemma. Let $\mathscr{M} \in \mathscr{R} \mathscr{M}_{o d}$, and put $M=\Gamma(X, \mathscr{M})$. Let $\rho_{\alpha}: M \rightarrow M_{\alpha}=$ $\Gamma\left(U_{\alpha}, \mathscr{M}\right)$ be the restriction map. Then $M_{\alpha} / \rho_{\alpha}(M)$ is in $\mathscr{T}_{\alpha}$ (that is, $R_{\alpha}$ $\left.\otimes_{R}\left(M_{\alpha} / \rho_{\alpha}(M)\right)=0\right)$.

Proof. We need to show that for each $m \in M_{\alpha}$, there exists $I \in \mathscr{F}_{\alpha}$ with $\operatorname{Im} \subset \rho_{\alpha}(M)$.

Let $m \in M_{\alpha}$. Put $m_{\beta}=\rho_{\alpha \beta}(m) \in M_{\alpha \beta}$. As $\mathscr{R}$ is quasicoherent, $R_{\alpha \beta} \otimes_{R_{\beta}}$ $\left(M_{\alpha \beta} / \rho_{\alpha \beta}\left(M_{\beta}\right)\right)=0$, so by Lemma $2.7, R_{\alpha} \otimes_{R}\left(M_{\alpha \beta} / \rho_{\alpha \beta}\left(M_{\beta}\right)\right)=0$. So for each $\beta$, there exists $I_{\beta} \in \mathscr{F}_{\alpha}$ with $I_{\beta} m_{\beta} \subset \rho_{\alpha \beta}\left(M_{\beta}\right)$. Put $J=\bigcap_{\beta} I_{\beta} \in \mathscr{F}_{\alpha}$. If $y \in J$ we can pick for each $\beta$ an element $m_{\beta}^{\prime} \in M_{\beta}$ with $y m_{\beta}=\rho_{\alpha \beta}\left(m_{\beta}^{\prime}\right)=\left.m_{\beta}^{\prime}\right|_{U_{\alpha \beta}}$. Notice that $\left.m_{\beta}^{\prime}\right|_{U_{\alpha \beta \gamma}}$ $=\left.y m_{\beta}\right|_{U_{\alpha \beta \gamma}}=\left.y m\right|_{U_{\alpha \beta \gamma}}$ so the elements $\left.m_{\beta}^{\prime}\right|_{U_{\beta \gamma}}$ and $\left.m_{\gamma}^{\prime}\right|_{U_{\beta \gamma}}$ have the same restriction to $U_{\alpha \beta \gamma}$. In other words $\left(\left.m_{\beta}^{\prime}\right|_{U_{\beta \gamma}}-\left.m_{\gamma}^{\prime}\right|_{U_{\beta \gamma}}\right) \in M_{\beta \gamma}$ restricts to zero on $U_{\alpha \beta \gamma}$, and hence this element is in the kernel of $\rho_{\alpha \beta \gamma}: M_{\beta \gamma} \rightarrow M_{\alpha \beta \gamma}$. But this kernel is $\tau_{\alpha}\left(M_{\beta \gamma}\right)$ so for each pair $(\beta, \gamma)$ there exists $I_{\beta \gamma} \in \mathscr{F}_{\alpha}$ with $I_{\beta \gamma}\left(\left.m_{\beta}^{\prime}\right|_{U_{\beta \gamma}}-\left.m_{\gamma}^{\prime}\right|_{U_{\beta \gamma}}\right)=0$. Put $J^{\prime}=$ $\bigcap_{\beta, \gamma} I_{\beta \gamma} \in \mathscr{F}_{\alpha}$. If $z \in J^{\prime}$, then $\left.z m_{\beta}^{\prime}\right|_{U_{\beta \gamma}}=\left.z m_{\gamma}^{\prime}\right|_{U_{\beta \gamma}}$, so the elements $z m_{\beta}^{\prime}$ glue to give a global section; that is, there exists $\frac{\beta \gamma}{m} \in M$ with $\left.\bar{m}\right|_{U_{\beta}}=z m_{\beta}^{\prime}$ for all $\beta$. By definition, $m_{\alpha}=m$, so $m_{\alpha}^{\prime}=y m_{\alpha}=y m$. Hence $\left.\bar{m}\right|_{U_{\alpha}}=z y m$ and $z y m \in \rho_{\alpha}(M)$. As $z \in J^{\prime}$ was arbitrary, $J^{\prime} y m \in \rho_{\alpha}(M)$.

The choice of $J^{\prime}$ depended on $y$. Pick elements $y_{1}, \ldots, y_{s}$ which generate $J$ and pick corresponding left ideals $J_{1}^{\prime}, \ldots, J_{s}^{\prime}$. Put $J^{\prime \prime}=J_{1}^{\prime} \cap \cdots \cap J_{s}^{\prime}$. We still have $J^{\prime \prime} \in \mathscr{F}_{\alpha}$ and $J_{i}^{\prime \prime} m \in \rho_{\alpha}(M)$ for $i=1, \ldots, s$. Hence if $I=J^{\prime \prime} y_{1}+\cdots+J^{\prime \prime} y_{s}$, then $\operatorname{Im} \in \rho_{\alpha}(M)$.

It remains to show that $I \in \mathscr{F}_{\alpha}$. As $R / J \in \mathscr{T}_{\alpha}$, it is enough to show that $J / I \in \mathscr{T}_{\alpha}$. As $J$ is generated by the $y_{i}$, it is enough to show that each $R y_{i}+I / I \in \mathscr{T}_{\alpha}$. But this module is a homomorphic image of $R / J^{\prime \prime}$ which is itself in $\mathscr{T}_{\alpha}$.

2.10. Proposition (Notation as In Lemma 2.8). The restriction map $\rho_{\alpha}: M \rightarrow M_{\alpha}$ extends to an isomorphism $\phi: R_{\alpha} \otimes_{R} M \rightarrow M_{\alpha}$.

Proof. Let $\phi$ denote the canonical extension of $\rho_{\alpha}$; that is $\phi(r \otimes m)=r \rho_{\alpha}(m)$ for $r \in R_{\alpha}, m \in M$. The diagram

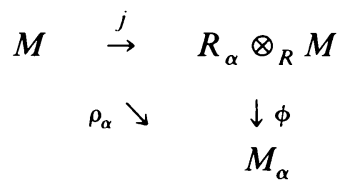

commutes, where $j(m)=1 \otimes m$.

As $j(M)$ is an essential submodule of $R_{\alpha} \otimes_{R} M$, if $\operatorname{ker} \phi \neq 0$, then $\operatorname{ker} \phi \cap j(M)$ $\neq 0$. Pick $m \in M$ with $j(m) \neq 0$ but $\phi j(m)=0$. Then $\rho_{\alpha}(m)=0$ but $j(m) \neq 0$. This is a contradiction as $\operatorname{ker} \rho_{\alpha}=\tau_{\alpha}(M)=\operatorname{ker} j$ by Lemma 2.8 . Hence $\phi$ is injective.

Let $m \in M_{\alpha}$. By Lemma 2.9, there exists $I \in \mathscr{F}_{\alpha}$ with $\operatorname{Im} \subset \rho_{\alpha}(M)$. But $m \in R_{\alpha} m$ $=R_{\alpha} \operatorname{Im} \subset R_{\alpha} \rho_{\alpha}(M)=i m \phi$. Hence $\phi$ is surjective.

2.11. Corollary. Let $\mathscr{M} \in \mathscr{R}-\mathscr{M}$ od and put $M=\Gamma(X, \mathscr{M})$. Then $\mathscr{M} \simeq \mathscr{R} \otimes_{R} M$.

Proof. If $U \subset X$ is any open affine subset, the restriction map $M \rightarrow M_{U}=$ $\Gamma(U, \mathscr{M})$ extends to a map $R_{U} \otimes_{R} M \rightarrow M_{U}$. Hence there is a morphism of presheaves $\mathscr{R} \otimes_{R} M \rightarrow \mathscr{M}$, and so a morphism of sheaves $\mathscr{R} \otimes_{R} M \rightarrow \mathscr{M}$. 
Because $\mathscr{R}$ is quasicoherent, $\left.\left.\mathscr{R} \otimes_{R} M\right|_{U_{\alpha}} \simeq \mathscr{R}\right|_{U_{\alpha}} \otimes_{R} M$, and so for any open affine $V \subset U_{\alpha}, \Gamma\left(V, \mathscr{R} \otimes_{R} M\right)=R_{V} \otimes_{R} M$. A consequence of the previous proposition is that if $V \subset U_{\alpha}$ is open affine then the natural map $R_{V} \otimes_{R} M \rightarrow M_{V}=\Gamma(V, \mathscr{M})$ is an isomorphism. Hence the morphism $\mathscr{R} \otimes_{R} M \rightarrow \mathscr{M}$ induces isomorphisms on the stalks, and so is an isomorphism itself.

In other words, each $\mathscr{M} \in \mathscr{R}=\mathscr{M} o d$ is generated by its global sections.

\subsection{Proposition. Let $N$ be an $R$-module and put $\mathscr{M}=\mathscr{R} \otimes_{R} N$. Then $\Gamma(X, \mathscr{M})=$} $N$.

Proof. Put $M=\Gamma(X, \mathscr{M})$. First we show there exists an injective map from $N$ into $M$.

Define a map $N \rightarrow M$ by associating with each $n \in N$ the global section $s$ of $\mathscr{M}$ such that $s(x)=1 \otimes n \in R_{x} \otimes_{R} N$ for each $x \in X$, where $R_{x}$ is the stalk of $\mathscr{R}$ at $x$. Pick $U_{\alpha}$ containing $x$. As $\mathscr{R}$ is quasicoherent, $R_{x}=A_{x} \otimes_{A_{\alpha}} R_{\alpha}$ and $R_{x} \otimes_{R} N=A_{x}$ $\otimes_{A_{\alpha}} R_{\alpha} \otimes_{R} N$. If $s=0$ then $1 \otimes 1 \otimes n \in A_{x} \otimes_{A_{\alpha}} R_{\alpha} \otimes_{R} N$ is zero for all $x \in U_{\alpha}$. But as $\prod_{x \in U_{\alpha}} A_{x}$ is faithfully flat over $A_{\alpha}, 1 \otimes n$ considered as an element of $R_{\alpha} \otimes_{R} N$ is zero. Hence $1 \otimes n \in R_{\alpha} \otimes_{R} N$ is zero for all $\alpha$. But $\oplus R_{\alpha}$ is faithfully flat over $R$ so $n=0$.

As $R_{\alpha}$ is flat over $R$, the map above extends to an injective map $R_{\alpha} \otimes_{R} N \rightarrow R_{\alpha}$ $\otimes_{R} M$. As $\mathscr{R}$ is quasicoherent, the restriction of the presheaf $\mathscr{R} \otimes_{R} N$ to $U_{\alpha}$ is a sheaf, and hence $\Gamma\left(U_{\alpha}, \mathscr{M}\right)=R_{\alpha} \otimes_{R} N$. So the map $R_{\alpha} \otimes_{R} N \rightarrow R_{\alpha} \otimes_{R} M$ is a bijection by Proposition 2.10. In particular, as $R_{\alpha}$ is flat, $R_{\alpha} \otimes_{R}(M / N)=0$. But this is true for all $\alpha$, so the faithful flatness of $\oplus R_{\alpha}$ implies that $M / N=0$. Hence $M=N$ as required.

It should be remarked that the quasicoherence of $\mathscr{R}$ implies that $\mathscr{R} \otimes_{R} N$ is also quasicoherent.

2.13. Proof of THE TheOREM. The Theorem is an immediate consequence of Corollary 2.11 and Proposition 2.12. We have shown that the functors $M \rightarrow \mathscr{R} \otimes_{R} M$ and $\mathscr{M} \rightarrow \Gamma(X, \mathscr{M})$ are mutually inverse to one another when considered as maps from the objects of one category to the objects of the the other category. It is immediate that an $R$-module homomorphism $M \rightarrow M^{\prime}$ extends to a morphism of presheaves $\mathscr{R} \otimes_{R} M \rightarrow \mathscr{R} \otimes_{R} M^{\prime}$, and hence gives a morphism of sheaves $\mathscr{M} \rightarrow \mathscr{M}^{\prime}$. Likewise a morphism $\mathscr{M} \rightarrow \mathscr{M}^{\prime}$ gives a morphism $\Gamma(X, \mathscr{M}) \rightarrow \Gamma\left(X, \mathscr{M}^{\prime}\right)$. It follows that the two functors are inverse to one another when considered as maps on the morphisms.

2.14. In [BB] the equivalence of categories is proved by showing that every $\mathscr{M} \in \mathscr{D}_{\lambda}=\mathscr{M}_{o d}$ is generated by its global sections and that $H^{i}(X, \mathscr{M})=0$ for $i>0$. We have been unable to show under the hypotheses of $\S 1.2$ that $H^{i}(X, \mathscr{M})=0$ for $i>0$. However, if we assume that $\mathscr{R}$ is flat over $X$ (that is, $R_{x}$ is a flat $A_{x}$-module for each $x \in X$ ) then we have the following result. We remark first that it is easy to show each $\mathscr{D}_{\lambda}$ satisfies this condition (the stalk of $\mathscr{D}_{\lambda}$ at $x$ is just the ring of differential operators on the regular local ring $A_{x}$, and the ring of differential operators on $A_{x}$ is free as an $A_{x}$-module). 
Proposition. If $\mathscr{R}$ is flat over $X$, then each $\mathscr{M} \in \mathscr{R}-\mathscr{M}$ od satisfies $H^{i}(X, \mathscr{M})=0$ for $i>0$.

Proof. Put $M=\Gamma(X, \mathscr{M})$ and take an injective resolution $0 \rightarrow M \rightarrow E_{1} \rightarrow E_{2}$ $\rightarrow \cdots$ of the $R$-module $M$. By the equivalence of categories $0 \rightarrow \mathscr{M} \rightarrow \mathscr{R} \otimes_{R} E_{1} \rightarrow$ $\mathscr{R} \otimes_{R} E_{2} \rightarrow \cdots$ is an injective resolution in the category $\mathscr{R} \mathscr{M} \circ d$

Claim. If $E$ is an injective $R$-module, then $\mathscr{R} \otimes_{R} E$ is injective in the category of sheaves of quasicoherent $\mathcal{O}$-modules.

Let $0 \rightarrow B^{\prime} \rightarrow(S H$ be an exact sequence of quasicoherent $\mathcal{O}$-modules and suppose $f$ :

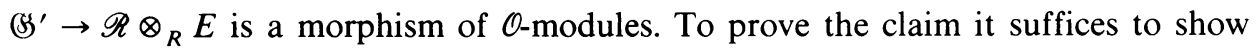
that there exists a morphism $g:\left(\mathfrak{H} \rightarrow \mathscr{R} \otimes_{R} E\right.$ whose restriction to (H') is $f$.

The natural map $\mathscr{R} \otimes_{\mathcal{O}} \mathscr{S}^{\prime} \rightarrow \mathscr{R} \otimes_{\mathcal{O}}$ (S) is injective on stalks as each $R_{x}$ is a flat $A_{x}$-module, so is an injective morphism of $\mathcal{O}$-modules. A tensor product of quasicoherent $\mathcal{O}$-modules is again quasicoherent, so both these modules are in $\mathscr{R}-\mathscr{M}$ od.

The morphism $f$ induces a morphism $\bar{f}: \mathscr{R} \otimes_{\mathcal{O}} \mathscr{S S}^{\prime} \rightarrow \mathscr{R} \otimes_{R} E$. Now as $\mathscr{R} \otimes_{R} E$ is injective in $\mathscr{R} \mathscr{M} \circ d, \bar{f}$ extends to a map $\bar{g}: \mathscr{R} \otimes_{\mathcal{O}}\left(\mathfrak{S} \rightarrow \mathscr{R} \otimes_{R} E\right.$. The map $g$ is then the composition (S) $\rightarrow \mathscr{R} \otimes(S) \rightarrow \mathscr{R} \otimes E$. This proves the claim.

Now, by [H, III, Example 3.6], $\mathscr{R} \otimes_{R} E$ is flasque so we may calculate cohomology from the sequence $0 \rightarrow \mathscr{M} \rightarrow \mathscr{R} \otimes E_{1} \rightarrow \mathscr{R} \otimes E_{2} \rightarrow \cdots$ after applying $\Gamma(X,-)$. But, by the equivalence of categories, this gives the exact sequence $0 \rightarrow M \rightarrow E_{1} \rightarrow E_{2}$ $\rightarrow \cdots$. Hence $H^{i}(X, \mathscr{M})=0$ for $i>0$.

2.15. As remarked in $\S 1.3$, condition (iii) of the assumptions in $\S 1.2$ follows from the equivalence of categories. A precise statement of this is the following

Proposition. Suppose that $\mathscr{R}$ satisfies conditions (i) and (ii) of $\S 1.2$, and that there is an equivalence between the categories $\mathscr{R}-\mathcal{M}$ od and $R$-Mod given by the mutually inverse functors $M \rightarrow \mathscr{R} \otimes_{R} M, \mathscr{M} \rightarrow \Gamma(X, \mathscr{M})$. Then condition (iii) of $\S 1.2$ also holds.

Proof. First we show that if $U \subset X$ is open affine, then $R_{U}$ is flat as a right $R$-module.

Let $0 \rightarrow M_{1} \rightarrow M_{2}$ be an exact sequence of $R$-modules. Put $\mathscr{M}_{i}=\mathscr{R} \otimes_{R} M_{i}$. The assumption implies that $0 \rightarrow \mathscr{M}_{1} \rightarrow \mathscr{M}_{2}$ is exact in $\mathscr{R}-\mathscr{M} o d$. Hence $0 \rightarrow \Gamma\left(U, \mathscr{M}_{1}\right)$ $\rightarrow \Gamma\left(U, \mathscr{M}_{2}\right)$ is an exact sequence of $R_{U}$-modules. But, as $\mathscr{R}$ is quasicoherent and $U$ is open affine, $\Gamma\left(U, \mathscr{M}_{i}\right)=R_{U} \otimes_{R} M_{i}$ (this is because the presheaf $\left.\mathscr{R} \otimes M\right|_{U}$ is already a sheaf over $U$ ). Hence $0 \rightarrow R_{U} \otimes_{R} M_{1} \rightarrow R_{U} \otimes_{R} M_{2}$ is exact, and $R_{U}$ is flat.

Suppose $\left(U_{\alpha}\right)$ is any open affine cover for $X$. Put $R_{\alpha}=\Gamma\left(U_{\alpha}, \mathscr{R}\right)$ and suppose $M$ is an $R$-module with $R_{\alpha} \otimes_{R} M=0$ for all $\alpha$. As just remarked, $R_{\alpha} \otimes_{R} M=$ $\Gamma\left(U_{\alpha}, \mathscr{R} \otimes M\right)$. So $\mathscr{R} \otimes M$ is locally zero, hence globally zero. The equivalence of categories implies that $M=\Gamma\left(X, \mathscr{R} \otimes_{R} M\right)$ is also zero. Hence $\oplus R_{\alpha}$ is faithfully flat.

In the proof of the proposition the choice of the $U_{\alpha}$ was arbitrary, so we have

COROLLARY. If $\mathscr{R}$ satisfies the conditions of $\$ 1.2$, then condition (iii) will hold for any open affine cover $\left(U_{\alpha}\right)$ of $X$. 


\section{Comments and remarks.}

3.1. We adopt here the notation of [BB]. The underlying topological space of the sheaf $\mathscr{D}_{\lambda}$ is $X=G / B$, the flag variety of the connected complex semisimple Lie group corresponding to the complex semisimple Lie algebra $g$. The cover $\left(U_{\alpha}\right)$ in this case could be obtained by taking the translates of the large Bruhat cell under the action of the Weyl group; that is each $U_{\alpha}=w B w_{0} B$ for some $w \in W$, the Weyl group, where $w_{0}$ is the long element of $W$. Each $R_{\alpha}$ is isomorphic to the Weyl algebra $A_{n}$, where $n=\operatorname{dim} X$. In [HS] it was shown that the restriction map $D_{\lambda}=\Gamma\left(X, \mathscr{D}_{\lambda}\right)$ $\rightarrow \Gamma\left(B w_{0} B, \mathscr{D}_{\lambda}\right)=A$ is (up to an automorphism of $\mathbf{g}$ and an automorphism of $A$ ) the Conze embedding $D_{w_{0} \lambda} \rightarrow A$ obtained through the action of $\mathbf{g}$ on the Verma module $M\left(w_{0} \lambda\right)$. In order to establish (iii) directly one would need to show (among other things) that the embedding $D_{w_{0} \lambda} \rightarrow A$ makes $A$ flat as a right $D_{w_{0} \lambda}$-module.

It is shown in [JS] for $\lambda$ dominant regular that $A$ is the union of the right $D_{w_{0} \lambda}$-modules $L\left(w_{0} \lambda, w_{0} \mu\right)$ (of g-finite maps from the Verma module $M\left(w_{0} \lambda\right)$ to $\left.M\left(w_{0} \mu\right)\right)$, where the union is taken over all dominant regular $\mu$ such that $\mu-\lambda$ is dominant integral. It already follows from [BG] that the $L\left(w_{0} \lambda, w_{0} \mu\right)$ are finitely generated projective right $D_{w_{0} \lambda}$-modules, and hence $A$ is flat as a right $D_{w_{0} \lambda}$-module.

3.2. The hypothesis that $X$ was irreducible was made only to avoid the possibility that some of the $U_{\alpha_{\mathrm{i}} \cdots \alpha_{n}}$ might be empty and therefore that some of the $R_{\alpha_{1} \cdots \alpha_{n}}$ might be zero. If one assumes that $X$ has a finite number of irreducible components $X_{i}$, and the hypotheses in $\$ 1.2$ are adjusted so that $\left.\mathscr{R}\right|_{X_{i}}$ satisfies (i)-(iii), then one can still show that $R$-Mod and $\mathscr{R}-\mathscr{M}$ od are equivalent.

\section{REFERENCES}

[BB] A. Beilinson and J. N. Bernstein, Localisation de g-modules, C. R. Acad. Sci. Sér. A-B 292 (1981), 15-18.

[BG] J. N. Bernstein and S. I. Gelfand, Tensor products of finite and infinite dimensional representations of semi-simple Lie algebras, Compositio Math. 41 (1980), 245-285.

[G] P. Gabriel, Des categories abeliennes, Bull. Soc. Math. France 90 (1962), 323-448.

[H] R. Hartshorne, Algebraic geometry, Graduate Texts in Math., no. 52, Springer-Verlag, New York, 1977.

[HS] T. J. Hodges and S. P. Smith, Differential operators on the flag variety and the Conze embedding (preprint).

[JS] A. Joseph and J. T. Stafford, Modules of $\mathbf{k}$-finite vectors over semi-simple Lie algebras, Proc. London Math. Soc. (3) 49 (1984), 361-384.

[St] B. Stenstrom, Rings of quotients, Springer-Verlag, New York, 1975.

Department of Mathematics, University of Utah, Salt lake City, Utah 84112

Mathematics Institute, University of Warwick, Coventry CV4 7AL, ENGland (Current address of S. P. Smith)

Current address (T. J. Hodges): Department of Mathematics, University of Cincinnati, Cincinnati, Ohio 45221 